\title{
The perimeter generating function of punctured staircase polygons
}

\author{
Anthony J. Guttmann and Iwan Jensen \\ ARC Centre of Excellence for Mathematics and Statistics of Complex Systems, \\ Department of Mathematics and Statistics, The University of Melbourne, Victoria \\ 3010, Australia \\ E-mail: tonyg, I. Jensen@ms . unimelb.edu .au
}

\begin{abstract}
Using a simple transfer matrix approach we have derived very long series expansions for the perimeter generating function of punctured staircase polygons (staircase polygons with a single internal staircase hole). We find that all the terms in the generating function can be reproduced from a linear Fuchsian differential equation of order 8 . We perform an analysis of the properties of the differential equation.
\end{abstract}

Submitted to: J. Phys. A: Math. Gen.

PACS numbers: $05.50 .+\mathrm{q}, 05.70 . J \mathrm{~J}, 02.10 . \mathrm{Ox}$ 


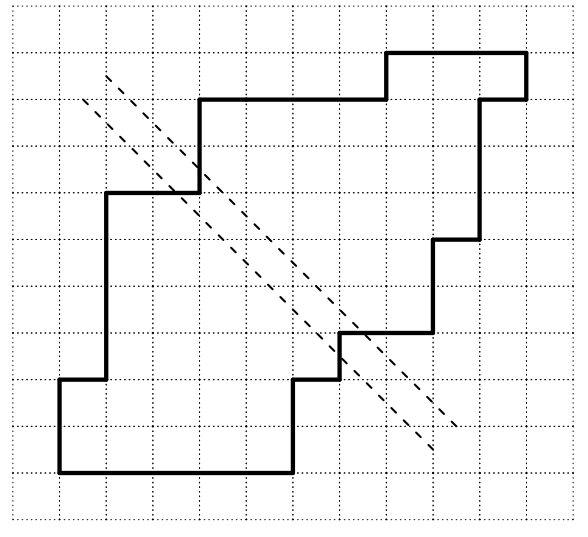

Staircase

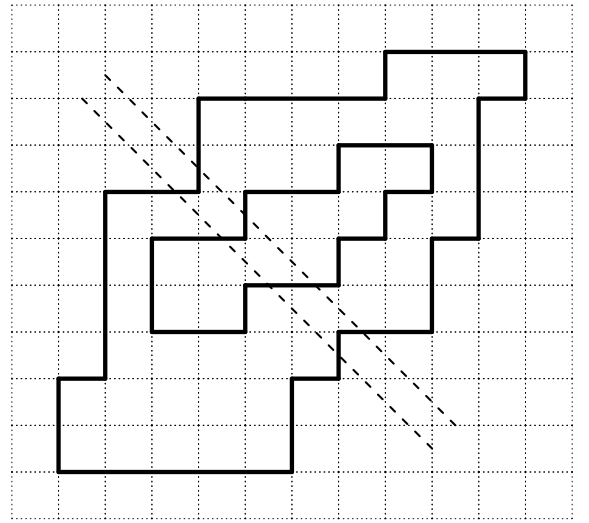

Punctured staircase

Figure 1. Examples of the types of polygons studied in this paper.

\section{Introduction}

A well-known long standing problem in combinatorics and statistical mechanics is to find the generating function for self-avoiding polygons (or walks) on a two-dimensional lattice, enumerated by perimeter. Recently, we have gained a greater understanding of the difficulty of this problem, as Rechnitzer [1] has proved that the (anisotropic) generating function for square lattice self-avoiding polygons is not differentiably finite [2], as had been conjectured earlier on numerical grounds [3]. That is to say, it cannot be expressed as the solution of an ordinary differential equation with polynomial coefficients. There are many simplifications of this problem that are solvable [4, but all the simpler models impose an effective directedness or equivalent constraint that reduces the problem, in essence, to a one-dimensional problem.

A staircase polygon can be viewed as the intersection of two directed walks starting at the origin, moving only to the right or up and terminating once the walks join at a vertex. It is well-known that the generating function for staircase polygons is

$$
P(x)=\frac{1-2 x-\sqrt{1-4 x}}{2} \propto(1-\mu x)^{2-\alpha},
$$

where the connective constant $\mu=4$ and the critical exponent $\alpha=3 / 2$. Punctured staircase polygons [5] are staircase polygons with internal holes which are also staircase polygons (the polygons are mutually- as well as self-avoiding). In [5] it was proved that the connective constant $\mu$ of $k$-punctured polygons (polygons with $k$ holes) is the same as the connective constant of unpunctured polygons. Numerical evidence clearly indicated that the critical exponent $\alpha$ increased by $3 / 2$ per puncture. The closely related model of punctured discs was considered in [6]. Punctured discs are counted by area and in this case it was proved that the critical exponent increases by 1 per puncture. Here we will study only the case with a single hole (see figure 11), and we will refer to these objects as punctured staircase polygons. The perimeter length of staircase polygons is 
even and thus the total perimeter (the outer perimeter plus the perimeter of the hole) is also even. We denote by $p_{n}$ the number of punctured staircase polygons of perimeter $2 n$. The results of [5] imply that the half-perimeter generating function has a simple pole at $x=x_{c}=1 / \mu=1 / 4$, though the analysis in [5] clearly indicated that the critical behaviour is more complicated than a simple algebraic singularity.

Recently we found that the perimeter generating function of three-choice polygons can be expressed as the solution of an 8th order linear ODE [7]. Similarly, in this paper we report on work which has led to an exact Fuchsian linear differential equation of order 8 apparently satisfied by the perimeter generating function, $\mathcal{P}(x)=\sum_{n \geq 0} p_{n} x^{n}$, for punctured staircase polygons (that is, $\mathcal{P}(x)$ is one of the solutions of the ODE, expanded around the origin). The first few terms in the generating function are

$$
\mathcal{P}(x)=x^{8}+12 x^{9}+94 x^{10}+604 x^{11}+3463 x^{12}+\cdots .
$$

Our analysis of the ODE shows that the dominant singular behaviour is

$$
\mathcal{P}(x) \sim \frac{A(x)}{(1-4 x)}+\frac{B(x)+C(x) \log (1-4 x)}{\sqrt{1-4 x}}+D(x)(1+4 x)^{13 / 2} .
$$

So in the notation used above, the generating function has a dominant singularity at $x=x_{c}=1 / \mu$ with exponent $\alpha=3$. This result confirms exactly the conjecture for the critical exponent [5] in the case of a single puncture and the quite complicated corrections at the critical point explains why the analysis in [5], based on a relatively short series, was so difficult.

It is also possible to express the generating function $\mathcal{P}(x)$ as a sum of $4 \times 4$ GesselViennot determinants [8]. This is clear from figure 2] where the enumeration of the lattice paths between the dotted lines is just the classical problem of 4 vicious walkers, and these must be joined to two vicious walkers to the left, and to two vicious walkers to the right. Then one must sum over different possible geometries. However the fact that the generating function is so expressible implies that it is differentiably finite [9].

Unfortunately we cannot readily bound the size of the underlying ODE, otherwise we could use this observation to provide a proof of our results. As it is, we originally generated the counts of punctured staircase polygons up to perimeter 502 (251 coefficients), and found what we believe to be the underlying ODE experimentally from the first 195 coefficients. The ODE then correctly predicts the next 56 coefficients. While the possibility that the underlying ODE is not the correct one is extraordinarily small, our procedure still does not constitute a proof of course. We have since extended the count beyond perimeter 800 and still all coefficients are predicted by our ODE.

\section{Computer enumeration}

The algorithm we use to count the number punctured staircase polygons is a modified version of the algorithm of Conway et al [10] for the enumeration of imperfect staircase polygons. The two problems are very similar and consequently there are only minor differences between the algorithms. A detailed description of the algorithm we used 


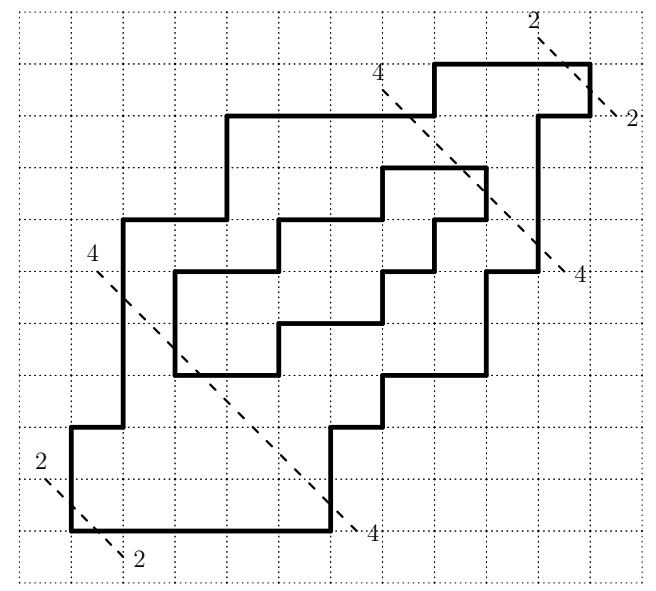

Figure 2. The decomposition of a punctured staircase polygon into a sequence of 2-4-2 vicious walkers, each expressible as a Gessel-Viennot determinant

to count imperfect staircase polygons can be found in [7. The algorithm is based on transfer matrix techniques. This entails bisecting the polygons by a line (as illustrated in figure 1) and enumerating the number of polygons by moving the line 'forward' one step at a time. Punctured staircase polygons start out as ordinary staircase polygons and the line bisects the polygon at two edges. Then at some vertex two additional directed walks (sharing the same starting point) are inserted between the two original walks. The line will thus intersect these polygon configurations at four edges (see figure 1). The only difference between the algorithm in [7] and the one used for this paper is in how the four directed walks intersected by the line are connected in order to produce a valid polygon. To produce a punctured staircase polygon we first connect the two innermost walks and then the two outermost walks are connected. Imperfect staircase polygons on the other hand are produced by connecting the first walk with the second walk and the third walks with the fourth walk. The updating rules used to count imperfect staircase polygons are given in [7] and are easily amended to count punctured staircase polygons bearing in mind the different 'closing' criteria outlined above.

We calculated the number of punctured staircase polygons up to perimeter 502. The integer coefficients become very large so the calculation was performed using modular arithmetic [1]. This involves performing the calculation modulo various prime numbers $p_{i}$ and then reconstructing the full integer coefficients at the end. We used primes of the form $p_{i}=2^{30}-r_{i}$, where $r_{i}$ are small positive integers, less than 1000 , chosen so that $p_{i}$ is prime, and $p_{i} \neq p_{j}$ unless $i=j .17$ primes were needed to represent the coefficients correctly. The calculation for each prime used about $200 \mathrm{Mb}$ of memory and about 8 minutes of CPU time on a $2.8 \mathrm{GHz}$ Xeon processor. Naturally we could have carried the calculation much further (and we have since done this) but as we shall demonstrate in the next section this number of coefficients more than sufficed to identify an exact differential equation satisfied by $\mathcal{P}(x)$. 


\section{The Fuchsian differential equations}

In recent papers Zenine et al [12, 13, 14] obtained the linear differential equations whose solutions give the 3 - and 4-particle contributions $\chi^{(3)}$ and $\chi^{(4)}$ to the Ising model susceptibility. In [7] we used their method to find a linear differential equation for threechoice polygons and in this paper we extend this work further to find a linear differential equation which has as a solution the generating function $\mathcal{P}(x)$ for punctured staircase polygons. We briefly outline the method here. Starting from a (long) series expansion for the function $\mathcal{P}(x)$ we look for a linear differential equation of order $m$ of the form

$$
\sum_{k=0}^{m} P_{k}(x) \frac{\mathrm{d}^{k}}{\mathrm{~d} x^{k}} \mathcal{P}(x)=0,
$$

such that $\mathcal{P}(x)$ is a solution to this homogeneous linear differential equation, where the $P_{k}(x)$ are polynomials. In order to make it as simple as possible we start by searching for a Fuchsian [15] equation. Such equations have only regular singular points. There are several reasons for searching for a Fuchsian equation, rather than a more general D-finite equation. Computationally the Fuchsian assumption simplifies the search for a solution. ¿From the general theory of Fuchsian [15] equations it follows that the degree of $P_{k}(x)$ is at most $n-m+k$ where $n$ is the degree of $P_{m}(x)$. To simplify matters further (reduce the order of the unknown polynomials) it is advantageous to explicitly assume that the origin and $x=x_{c}=1 / 4$ are regular singular points and set $P_{k}(x)=Q_{k}(x) S(x)^{k}$, where $S(x)=x(1-4 x)$. Thus when searching for a solution of Fuchsian type there are only two parameters, namely the order $m$ of the differential equation and the degree $q_{m}$ of the polynomial $Q_{m}(x)$. One may also argue, less precisely, that for "sensible" combinatorial models one would expect Fuchsian equations, as irregular singular points are characterized by explosive, super-exponential behaviour. Such behaviour is not normally characteristic of combinatorial problems arising from statistical mechanics. The point at infinity may be an exception to this somewhat imprecise observation.

We then search systematically for solutions by varying $m$ and $q_{m}$. In this way we first found a solution with $m=10$ and $q_{m}=11$, which required the determination of $L=195$ unknown coefficients. We have 251 terms in the half-perimeter series and thus have 56 additional terms with which to check the correctness of our solution. Having found this solution we then turned the ODE into a recurrence relation and used this to generate more series terms in order to search for a lower order Fuchsian equation. The lowest order equation we found was eighth order and with $q_{m}=27$, which requires the determination of $L=294$ unknown coefficients. Thus from our original 251 term series we could not have found this $8^{\text {th }}$ order solution since we did not have enough terms to determine all the unknown coefficients in the ODE. This raises the question as to whether perhaps there is an ODE of lower order than 8 that generates the coefficients? The short answer to this is no. Further study of our differential operator revealed that it can be factorised. In fact we found a factorization into three first-order linear operators, a second order and a third order. The generating function is a solution of the $8^{\text {th }}$ order 
Table 1. Critical exponents for the regular singular points of the Fuchsian differential equation satisfied by $\mathcal{P}(x)$.

\begin{tabular}{ll}
\hline Singularity & Exponents \\
\hline$x=0$ & $-1,0,0,0,1,2,3,8$ \\
$x=1 / 4$ & $-1,-1 / 2,-1 / 2,1 / 2,1,3 / 2,2,3$ \\
$x=-1 / 4$ & $0,1,2,3,4,5,6,13 / 2$ \\
$x= \pm \mathrm{i} / 2$ & $0,1,2,3,4,5,6,13 / 2$ \\
$1+x+7 x^{2}=0$ & $0,1,2,2,3,4,5,6$ \\
$1 / x=0$ & $-2,-3 / 2,-1,-1,-1 / 2,1 / 2,3 / 2,5 / 2$ \\
$Q_{8}(x)=0$ & $0,1,2,3,4,5,6,8$ \\
\hline
\end{tabular}

operator, not of any of the smaller factors.

The (half)-perimeter generating function $\mathcal{P}(x)$ for punctured staircase polygons is a solution to the linear differential equation of order 8

$$
\sum_{k=0}^{8} P_{n}(x) \frac{\mathrm{d}^{k}}{\mathrm{~d} x^{k}} \mathcal{P}(x)=0
$$

with

$$
\begin{array}{ll}
P_{8}(x)=x^{4}(1-4 x)^{8}(1+4 x)\left(1+4 x^{2}\right)\left(1+x+7 x^{2}\right) Q_{8}(x) . \\
P_{7}(x)=x^{3}(1-4 x)^{7} Q_{7}(x), & P_{6}(x)=2 x^{2}(1-4 x)^{6} Q_{6}(x), \\
P_{5}(x)=6 x(1-4 x)^{5} Q_{5}(x), & P_{4}(x)=120(1-4 x)^{4} Q_{4}(x), \\
P_{3}(x)=120(1-4 x)^{3} Q_{3}(x), & P_{2}(x)=720(1-4 x)^{2} Q_{2}(x), \\
P_{1}(x)=720(1-4 x) Q_{1}(x), & P_{0}(x)=2880 Q_{0}(x),
\end{array}
$$

where $Q_{8}(x), Q_{7}(x), \ldots, Q_{0}(x)$, are polynomials of degree $22,28,29,30,31,31,31,31$, and 31, respectively. The polynomials are listed in Appendix A.

The singular points of the differential equation are given by the roots of $P_{8}(x)$. One can easily check that all the singularities (including $x=\infty$ ) are regular singular points so equation (3) is indeed of the Fuchsian type. It is thus possible using the method of Frobenius to obtain from the indicial equation the critical exponents at the singular points. These are listed in Table 1

We shall now consider the local solutions to the differential equation around each singularity. Recall that in general it is known [16, 15] that if the indicial equation yields $k$ critical exponents which differ by an integer, then the local solutions may contain logarithmic terms up to $\log ^{k-1}$. However, for the Fuchsian equation (31) only multiple roots of the indicial equation give rise to logarithmic terms in the local solution around a given singularity, so that a root of multiplicity $k$ gives rise to logarithmic terms up to $\log ^{k-1}$. In particular this means that near any of the 22 roots of $Q_{8}(x)$ the local solutions have no logarithmic terms and the solutions are thus analytic since all the exponents are positive integers. The roots of $Q_{8}$ are thus apparent singularities [16, 15] of the 
Fuchsian equation (3). We briefly mention that as in our earlier study [7] we can find a solution of order 14 of the same form as (3) but with $Q_{14}(x)$ being just a constant. So at this order none of the roots of $Q_{8}(x)$ appear. Clearly any real singularity of the system cannot be made to vanish and so we conclude that the 22 roots of $Q_{8}$ must indeed be apparent singularities.

Assuming that only repeated roots give rise to log-terms, and thus that a sequence of positive integers give rise to analytic terms, then near the physical critical point $x=x_{c}=1 / 4=1 / \mu$ we expect the singular behaviour

$$
\mathcal{P}(x) \sim \frac{A(x)}{(1-4 x)}+\frac{B(x)+C(x) \log (1-4 x)}{\sqrt{1-4 x}},
$$

where $A(x), B(x)$ and $C(x)$ are analytic in a neighbourhood of $x_{c}$. Note that the terms associated with the exponents $1 / 2$ and $3 / 2$ become part of the analytic correction to the $(1-4 x)^{-1 / 2}$ term. Near the singularity on the negative $x$-axis, $x=x_{-}=-1 / 4$ we expect the singular behaviour

$$
\mathcal{P}(x) \sim D(x)(1+4 x)^{13 / 2},
$$

where again $D(x)$ is analytic near $x_{-}$. We expect similar behaviour near the pair of singularities $x= \pm \mathrm{i} / 2$, and finally at the roots of $1+x+7 x^{2}$ we expect the behaviour $E(x)\left(1+x+7 x^{2}\right)^{2} \log \left(1+x+7 x^{2}\right)$.

We can simplify the $8^{\text {th }}$ order differential operator found above. We first found three very simple solutions of the ODE, each corresponding to an order one differential operator,

$$
\begin{gathered}
F_{1}(x)=1-4 x, \\
F_{2}(x)=\frac{1-4 x-4 x^{3}}{1-4 x},
\end{gathered}
$$

and

$$
F_{3}(x)=\frac{9-34 x+14 x^{2}}{\sqrt{1-4 x}} .
$$

The existense of these three linearly independent solutions implies that we can find three first order operators, which we denote by $L_{i}^{(1)}$, with $i=1,2,3$, such that the original 8'th order differential operator can be written as $L^{(8)}=L^{(5)} L_{1}^{(1)} L_{2}^{(1)} L_{3}^{(1)}$, where $L^{(5)}$ is a fifth order differential operator. We further found that this latter operator is decomposable as $L^{(5)}=L^{(3)} L^{(2)}$. This then allows one, in principle, to write down the form of the $8 \times 8$ matrix representing the differential Galois group of $L^{(8)}$, in an appropriate global solution basis. To determine the asymptotics one would need to calculate non-local connection matrices between solutions at different points. This is a huge task for such a large differential operator. Instead, we have developed a numerical technique that avoids all these difficulties, which we describe below.

To standardise our asymptotic analysis, we assume that the critical point is at 1 . The growth constant of punctured staircase staircase polygons is 4 , so we normalise the series by considering the new series with coefficients $r_{n}$, defined by $r_{n}=p_{n+8} / 4^{n}$. Thus the generating function we study is $\mathcal{R}(y)=\sum_{n \geq 0} r_{n} y^{n}=1+3 y+5.875 y^{2}+\cdots$. Using 
the recurrence relations for $p_{n}$ (derived from the ODE) it is easy and fast to generate many more terms $r_{n}$. We generated the first 100000 terms and saved them as floats with 500 digit accuracy (this calculation took less than 15 minutes). ¿From equations (5) and (6) it follows that the asymptotic form of the coefficients is

$$
\left[y^{n}\right] \mathcal{R}(y)=r_{n}=\sum_{i \geq 0}\left(\frac{\tilde{a}_{i}}{n^{i}}+\frac{\tilde{b}_{i} \log n+\tilde{c}_{i}}{n^{i+1 / 2}}+(-1)^{n}\left(\frac{\tilde{d}_{i}}{n^{15 / 2+i}}\right)\right)+\mathrm{O}\left(\lambda^{-n}\right) .
$$

Any contributions from the other singularities are exponentially suppressed since their norm (in the scaled variable $y=x / 4$ ) exceeds 1 .

Estimates for the amplitudes were obtained by fitting $r_{n}$ to the form given above using an increasing number of amplitudes. 'Experimentally' we find we need about the same total number of terms at $x_{c}$ and $-x_{c}$. So in the fits we used the terms with amplitudes $\tilde{a}_{i}, \tilde{b}_{i}$, and $\tilde{c}_{i}, i=0, \ldots, K$ and $\tilde{d}_{i}, i=0, \ldots, 3 K$. Going only to $K$ with the $\tilde{d}_{i}$ amplitudes results in much poorer convergence and going beyond $3 K$ leads to no improvement. For a given $K$ we thus have to estimate $6 K+4$ unknown amplitudes. So we use the last $6 K+4$ terms $r_{n}$ with $n$ ranging from 100000 to $100000-6 K-3$ and solve the resulting $6 K+4$ system of linear equations. We can also add extra terms to the asymptotic form and check what happens to the amplitudes of the new terms. If these amplitudes are very small it is highly likely that the terms are not truly present (if the calculation could be done exactly these amplitudes would be zero).

Doing this we found that all the amplitudes $\tilde{a}_{i}$ appear to be zero except that $\tilde{a}_{0}=1024$, e.g., with $K=20$ we find that the estimates for the amplitudes $\tilde{a}_{1}<10^{-70}$, $\tilde{a}_{2}<10^{-60}, \tilde{a}_{3}<10^{-50}$, etc. So in all likelihood the amplitudes $\tilde{a}_{i}=0$ for $i>0$. This then leads us to the refined asymptotic form

$\left[y^{n}\right] \mathcal{R}(y)=r_{n}=1024\left(1+\frac{1}{\sqrt{n}} \sum_{i \geq 0}\left(\frac{b_{i} \log n+c_{i}}{n^{i}}+(-1)^{n}\left(\frac{d_{i}}{n^{7+i}}\right)\right)\right)+\mathrm{O}\left(\lambda^{-n}\right)$.

In fits to this form we then used the terms with amplitudes $b_{i}$, and $c_{i}, i=0, \ldots, K$ and $d_{i}, i=0, \ldots, 2 K$. For a given $K$ we thus have to estimate $4 K+3$ unknown amplitudes. We find that the amplitude estimates are fairly accurate up to around $2 K / 3$. We observed this by doing the calculation with $K=30$ and $K=40$ and then looking at the difference in the amplitude estimates. For $b_{0}$ and $c_{0}$ the difference is less than $10^{-120}$, while for $d_{0}$ the difference is less than $10^{-116}$. Each time we increase the amplitude index by 1 we lose around six significant digits in accuracy. With $i=18$ the differences are respectively around $10^{-14}$ and $10^{-11}$.

¿From our very long series it is possible to obtain accurate numerical estimates of many of the amplitudes $b_{i}, c_{i}$, and $d_{i}$, with a precision of more than 100 digits for the dominant amplitudes, shrinking to around 10 digits for the the case when $i=18$ (actually we could probably have pushed this further but there would be little point). In this way we found that $b_{0}=-\frac{6 \sqrt{3}}{\pi^{3 / 2}}, b_{1}=\frac{305}{4 \sqrt{3} \pi^{3 / 2}}, b_{2}=\frac{86123}{192 \sqrt{3} \pi^{3 / 2}}$, $c_{0}=1.55210340048879105374 \ldots$ and $d_{0}=\frac{48}{\pi^{3 / 2}}, d_{1}=-\frac{2610}{\pi^{3 / 2}}, d_{2}=\frac{640815}{8 \pi^{3 / 2}}, d_{3}=-\frac{116785575}{64 \pi^{3 / 2}}$, $d_{4}=\frac{70325480841}{2048 \pi^{3 / 2}}$, though we have not been able to identify $c_{0}$. These amplitudes are known to at least 100 digits accuracy. 
The excellent convergence is solid evidence (though naturally not a proof) that the assumptions leading to equation (7) are correct. Further evidence was obtained as follows: We have already argued that the terms of the form $1 / n^{i}, i>0$ are absent. We found similar results if we added terms like $\log n / n^{i}, \log ^{2} n / n^{i / 2}$ or additional $\log n$ terms at $y=-1$. So this fitting procedure provides convincing evidence that the asymptotic form (8), and thus the assumptions leading to this formula, are correct.

\section{Conclusion and Outlook}

We have developed an improved algorithm for enumerating punctured staircase polygons. The extended series, coupled with a search program that assumes the solution is a Fuchsian ODE, enabled us to discover the underlying ODE, which was of $10^{\text {th }}$ order. We did this without using 56 of the coefficients that we had generated. That is to say, 56 of the known coefficients were unused, and so their values provided a check on the solution. This leads us to believe that we have found the correct ODE, as it reproduces the known, unused coefficients. Subsequently we have extended this check to more than 200 unused coefficients. Further refinement allowed us to find an $8^{\text {th }}$ order ODE.

A numerical technique we have developed specifically for such problems then allowed us to find accurate numerical estimates for the amplitudes of the first several terms in the asymptotic form of the coefficients, and then to conjecture their exact value.

We have also initiated an investigation of the area generating function. We expect this to involve $q$-series, and thus far our investigations only lead us to believe that the area generating function $A(q)$ is of the form

$$
A(q)=(G(q)+H(q) \sqrt{1-q / \eta}) /\left[J_{0}(1,1, q)^{2}\right]
$$

where $J_{0}(x, y, q)$ is a $q$-generalisation of the Bessel function, and occurs, for example, in the solution of the problem of staircase polygons enumerated by perimeter [4. Here $q=\eta$ is the first zero of $J_{0}(1,1, q)$, and $G$ and $H$ are regular in the neighbourhood of $q=\eta$. The coefficients thus behave asymptotically as

$$
a_{n}=\left[q^{n}\right] A(q) \sim \text { const. } \eta^{-n} n
$$

In a subsequent publication we propose to investigate the area generating function more fully, and hopefully obtain more insight into the properties of the ODE we have found for the perimeter generating function.

Furthermore in work with C. Richard [17] we have conjectured the scaling function for punctured polygons with an arbitrary number of punctures. We briefly review the properties of the two-variable area-perimeter generating function for staircase polygons. Of special interest is the point $\left(x_{c}, 1\right)$ where two lines of singularities meet. The behaviour of the singular part of the generating function about $\left(x_{c}, 1\right)$ is expected to take the special form

$$
P(x, q) \sim P^{(r e g)}(x, q)+(1-q)^{\theta} F\left(\left(x_{c}-x\right)(1-q)^{-\phi}\right), \quad(x, q) \nearrow,
$$


where $F(s)$ is a scaling function of combined argument $s=\left(x_{c}-x\right)(1-q)^{-\phi}$, commonly assumed to be regular at the origin, and $\theta=1 / 3$ and $\phi=2 / 3$ are critical exponents. For staircase polygons, we have

$$
F(s)=\frac{1}{8} \frac{\mathrm{d}}{\mathrm{d} s} \log \operatorname{Ai}\left((4 \sqrt{2})^{\frac{2}{3}} s\right) .
$$

In [17] we assumed that the limit distribution by area of staircase polygons with $r$ punctures (of arbitrary size) is that of staircase polygons with $r$ holes of unit area. From this and knowledge of $F(s)$ we then obtained exact predictions for $r$ punctured staircase polygons by taking the $r$-th derivative w.r.t $q$ of $P(x, q)$. We then study the area-moment generating function, $P_{k}(x)=\sum_{m, n} n^{k} p_{m, n} x^{m}$, where $p_{m, n}$ is the number of polygons with perimeter $m$ and area $n$. In particular we find that the leading amplitudes $A_{k}^{(r)}$ of the perimeter generating function of the $k$-th area-moment are given by

$$
A_{k}^{(r)}=\frac{(k+r) ! x_{c}^{r} f_{k+r}}{r ! x_{c}^{\gamma_{k+r}} \Gamma\left(\gamma_{k+r}\right)}
$$

Here $f_{k+r}$ are amplitudes occuring in the asymptotic expansion of $P(x, q)$ (these are known exactly for punctured staircase polygons) while $\gamma_{k+r}=3(k+r) / 2-1 / 2$ are the critical exponents of the $k$ th area-moment of $r$ punctured polygons. These predictions have been confirmed for once punctured staircase polygons to a very high level of accuracy for moments up to $k=10$. The numerical analysis of the area-moments relied crucially on our knowledge of the singularity structure of the perimeter generating function as detailed in this paper.

\section{E-mail or WWW retrieval of series}

The series for the generating functions studied in this paper can be obtained via e-mail by sending a request to I.Jensen@ms.unimelb.edu.au or via the world wide web on the URL http://www.ms.unimelb.edu.au/ iwan// by following the instructions.

\section{Acknowledgments}

We would like to thank N Zenine and J-M Maillard for access to their Mathematica routines for identifying differential equations and useful advice about their use. We gratefully acknowledge financial support from the Australian Research Council.

\section{Appendix A. Polynomials $Q_{n}(x)$ for punctured staircase polygons}

$$
\begin{aligned}
Q_{8}(x)= & -90720+1255590 x-9538200 x^{2}+20394828 x^{3}-79106610 x^{4} \\
& +1223958687 x^{5}-2943232056 x^{6}+17470357067 x^{7}-189472079743 x^{8} \\
& +579172715823 x^{9}-1746461498616 x^{10}+8400325324610 x^{11} \\
& -1591154327260 x^{12}-111431714394808 x^{13}+315517552430480 x^{14}
\end{aligned}
$$




$$
\begin{aligned}
& -106489387477312 x^{15}-938487878760384 x^{16}+1628517397980288 x^{17} \\
& -2394531569420032 x^{18}+2966185168205312 x^{19} \\
& -170238270849024 x^{20}-699187344629760 x^{21}+295462090506240 x^{22} \\
& Q_{7}(x)=-1360800+23565780 x-167569290 x^{2}+478254996 x^{3}+641052858 x^{4} \\
& +14810951034 x^{5}-47034372339 x^{6}-166933659974 x^{7}-2552936187594 x^{8} \\
& +6447911404224 x^{9}+14253364474478 x^{10}+86598771199392 x^{11} \\
& +362131239586500 x^{12}-3860712252484892 x^{13}+8993313236994576 x^{14} \\
& -31235880957264960 x^{15}+46429326957124912 x^{16} \\
& +155905775680790304 x^{17}-807736441103822976 x^{18} \\
& +1835072857042276096 x^{19}-1278888252797142528 x^{20} \\
& -293981468599460352 x^{21}+14541716059525437440 x^{22} \\
& -26481815895022608384 x^{23}+22483566008412450816 x^{24} \\
& -35911819535956066304 x^{25}+3639680241277796352 x^{26} \\
& +7495959535363031040 x^{27}-3507725938490081280 x^{28} \\
& \begin{aligned}
Q_{6}(x)= & -1723680+69281730 x-787195710 x^{2}+4886678970 x^{3}-10726639974 x^{4} \\
& +11830409583 x^{5}-401281487235 x^{6}+343905413598 x^{7} \\
& +1858137414650 x^{8}+44092692217413 x^{9}-36740412036168 x^{10} \\
& -135298590380414 x^{11}-1279093006602396 x^{12}-10004750418032976 x^{13} \\
& +61536871579988144 x^{14}-216281351081049504 x^{15} \\
& +1050287576547538488 x^{16}-1795967175346626976 x^{17} \\
& -2572736181692580960 x^{18}+18017037664470796032 x^{19} \\
& -45232775265352713472 x^{20}+48709527110201501184 x^{21} \\
& +4770083118869915136 x^{22}-322327838255331590144 x^{23} \\
& +541571044899035842560 x^{24}-511926023257614434304 x^{25} \\
& +716375351150156644352 x^{26}-69659801950830723072 x^{27} \\
& -136551990333116252160 x^{28}+60094625512245166080 x^{29}
\end{aligned} \\
& Q_{5}(x)=1965600-6539400 x-358033410 x^{2}+4831433820 x^{3}-30915098190 x^{4} \\
& +60211846008 x^{5}-201764518161 x^{6}+2531858233470 x^{7} \\
& +1380416576424 x^{8}-20212314275250 x^{9}-61506470769366 x^{10} \\
& -477804842150324 x^{11}+608746761166938 x^{12}+483723642457152 x^{13} \\
& +60127368616743592 x^{14}-185780400624937008 x^{15}
\end{aligned}
$$




$$
\begin{aligned}
& +1165835175099337288 x^{16}-7175943616536571776 x^{17} \\
& +13745698284061066112 x^{18}+4948349174336379840 x^{19} \\
& -89453290124304769024 x^{20}+270104157697832561664 x^{21} \\
& -356324521463829808128 x^{22}-41862184650482117632 x^{23} \\
& +1845216328946812827648 x^{24}-2906213125616330383360 x^{25} \\
& +2943265956913569742848 x^{26}-3723507915329643413504 x^{27} \\
& +405249143061461336064 x^{28}+618215144006850969600 x^{29} \\
& -261821958729561538560 x^{30}
\end{aligned}
$$

$$
\begin{aligned}
Q_{4}(x)= & 241920-8017380 x+88351704 x^{2}-590355612 x^{3}+2409400818 x^{4} \\
& -8457027588 x^{5}+71232186468 x^{6}-288557341128 x^{7} \\
& +524905454055 x^{8}-5046532132734 x^{9}+28114089314043 x^{10} \\
& -164508486596467 x^{11}+869331744354740 x^{12}-2401501341116904 x^{13} \\
& +12275987679372578 x^{14}-50846889626226508 x^{15} \\
& +46258831828476364 x^{16}-147764159295056304 x^{17} \\
& +1375769527659995736 x^{18}-2625251094439093408 x^{19} \\
& -765792895039661984 x^{20}+22951686058011476032 x^{21} \\
& -85054223999548283904 x^{22}+126294091912315062016 x^{23} \\
& +19381267403906712064 x^{24}-566287434634380073984 x^{25} \\
& +849895463062111623168 x^{26}-892557255237919469568 x^{27} \\
& +1043719341871898804224 x^{28}-142670999896790335488 x^{29} \\
& -140350544778022354944 x^{30}+59234239904690995200 x^{31}
\end{aligned}
$$

$$
\begin{aligned}
Q_{3}(x)= & -4596480+112443660 x-1327020156 x^{2}+11580963786 x^{3}-76436209584 x^{4} \\
& +426159579924 x^{5}-2350462539072 x^{6}+11395385983233 x^{7} \\
& -44136036344190 x^{8}+145288111685523 x^{9}-559910802106640 x^{10} \\
& +3013037795053530 x^{11}-13499762948930634 x^{12} \\
& +50096716464628528 x^{13}-217987216302493908 x^{14} \\
& +853439326193439492 x^{15}-2363497210984795232 x^{16} \\
& +5096223845046539304 x^{17}-8508469151526998016 x^{18} \\
& +9581930085552894304 x^{19}-10241374665198721536 x^{20} \\
& -12641088914996048640 x^{21}+118651673978481267200 x^{22} \\
& -208768950136609496064 x^{23}-15400291418459486208 x^{24} \\
& +814317146169694152704 x^{25}-1202858442211165741056 x^{26}
\end{aligned}
$$


Punctured staircase polygons

$$
\begin{aligned}
& +1271933402411862171648 x^{27}-1406355411740766470144 x^{28} \\
& +251165051564655771648 x^{29}+137326949251639934976 x^{30} \\
& -61285928661166325760 x^{31}
\end{aligned}
$$

$$
\begin{aligned}
Q_{2}(x)= & 1209600-10784340 x+25225200 x^{2}-192390408 x^{3}+1497608946 x^{4} \\
& -3085618896 x^{5}+55270573062 x^{6}-674664767886 x^{7}+1891951243653 x^{8} \\
& +6937954472784 x^{9}-19443421819978 x^{10}-252270853719194 x^{11} \\
& +1421753108033868 x^{12}-2280488850916676 x^{13}-1040351739238056 x^{14} \\
& -1519080794794788 x^{15}+54144924827952720 x^{16} \\
& -143110935850986376 x^{17}-63031554528921744 x^{18} \\
& +1125126938486807936 x^{19}-2675665192031509504 x^{20} \\
& +3361130538055156224 x^{21}-2669659667713374208 x^{22} \\
& +1996890960732463104 x^{23}-4866848788151009280 x^{24} \\
& +3555378162093901824 x^{25}+3193922372633202688 x^{26} \\
& -2642707373157531648 x^{27}+2132642211038560256 x^{28} \\
& -3311881541411143680 x^{29}+1596569887904366592 x^{30} \\
& -264734033093591040 x^{31}
\end{aligned}
$$

$$
\begin{aligned}
Q_{1}(x)= & -725760+19969740 x-254689092 x^{2}+2329185726 x^{3}-17948325636 x^{4} \\
& +118028863386 x^{5}-679983561900 x^{6}+3637871524611 x^{7} \\
& -17150360490738 x^{8}+62088405193554 x^{9}-183555964459890 x^{10} \\
& +747009873725220 x^{11}-4106684548673028 x^{12}+18540613780587884 x^{13} \\
& -67936944600058776 x^{14}+247341581626824360 x^{15} \\
& -939866071520217104 x^{16}+3216462341735279616 x^{17} \\
& -8789133587934808704 x^{18}+17976423995943224576 x^{19} \\
& -26625353996773725696 x^{20}+29354499014436664320 x^{21} \\
& -26197184327864145920 x^{22}+20118012206750361600 x^{23} \\
& -11595016904008224768 x^{24}-12803308242930466816 x^{25} \\
& +49275320633035751424 x^{26}-49679788190366564352 x^{27} \\
& +31169615491025600512 x^{28}-29010025645678264320 x^{29} \\
& +12772559103234932736 x^{30}-2117872264748728320 x^{31}
\end{aligned}
$$

$$
Q_{0}(x)=Q_{1}(x)
$$




\section{References}

[1] Rechnitzer A 2003 Haruspicy and anisotropic generating functions Adv. Appl. Math. 30 228-257

[2] Stanley R P 1980 Differentiably finite power series Eur. J. Comb. 1 175-188

[3] Guttmann A J and Conway A R 2001 Square lattice self-avoiding walks and polygons Ann. Comb. 5 319-345

[4] Bousquet-Mélou M 1996 A method for the enumeration of various classes of column-convex polygons Disc. Math. 154 1-25

[5] Guttmann A J, Jensen I, Wong L H and Enting I G 2000 Punctured polygons and polyominoes on the square lattice J. Phys. A: Math. Gen. 33 1735-1764

[6] Janse van Rensburg E J and Whittington S G 1990 Punctured discs on the square lattice J. Phys. A: Math. Gen. 23 1287-1294

[7] Guttmann A J and Jensen I 2006 Fuchsian differential equation for the perimeter generating function of three-choice polygons Séminaire Lotharingien de Combinatoire 54 B54c. Preprint: math.CO/0506317

[8] Gessel I and Viennot X G 1989 Determinants, paths and plane partitions. Preprint at http://people.brandeis.edu/ gessel//

[9] Lipshitz L 1989 D-finite power series J. Algebra 122 353-373

[10] Conway A R, Guttmann A J and Delest M 1997 The number of three-choice polygons Mathl. Comput. Modelling 26 51-58

[11] Knuth D E 1969 Seminumerical Algorithms. The Art of Computer Programming, Vol 2. (Reading, Mass: Addison Wesley)

[12] Zenine N, Boukraa S, Hassani S and Maillard J M 2004 The Fuchsian differential equation of the square lattice Ising model $\chi^{(3)}$ susceptibility J. Phys. A: Math. Gen. 37 9651-9668

[13] Zenine N, Boukraa S, Hassani S and Maillard J M 2005 Square lattice Ising model susceptibility: series expansion method and differential equation for $\chi^{(3)}$ J. Phys. A: Math. Gen. 38 1875-1899

[14] Zenine N, Boukraa S, Hassani S and Maillard J M 2005 Ising model susceptibility: the Fuchsian differential equation for $\chi^{(4)}$ and its factorization properties J. Phys. A: Math. Gen. 384149 4173

[15] Ince E L 1927 Ordinary differential equations (London: Longmans, Green and Co. Ltd.)

[16] Forsyth A R 1902 Part III. Ordinary linear equations vol. IV of Theory of differential equations. (Cambridge: Cambridge University Press)

[17] Richard C, Jensen I and Guttmann A J 2006 Scaling function for punctured staircase and selfavoiding polygons in preparation 